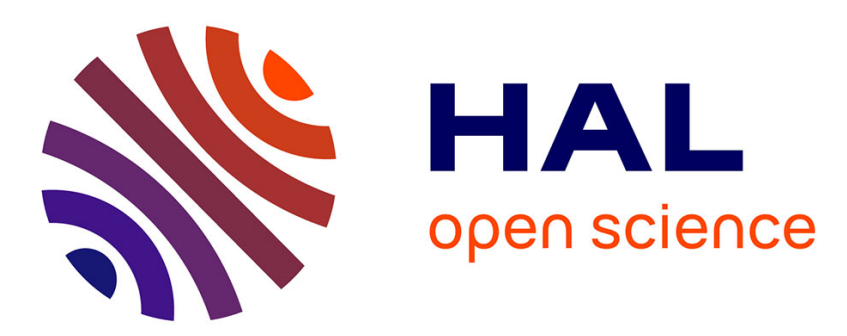

\title{
Facteurs de variation génétiques de la prise alimentaire chez le porc en croissance: le point des connaissances
}

F. Labroue

\section{To cite this version:}

F. Labroue. Facteurs de variation génétiques de la prise alimentaire chez le porc en croissance: le point des connaissances. Productions Animales, 1995, 8 (4), pp.239-250. hal-00896119

\section{HAL Id: hal-00896119 \\ https://hal.science/hal-00896119}

Submitted on 1 Jan 1995

HAL is a multi-disciplinary open access archive for the deposit and dissemination of scientific research documents, whether they are published or not. The documents may come from teaching and research institutions in France or abroad, or from public or private research centers.
L'archive ouverte pluridisciplinaire HAL, est destinée au dépôt et à la diffusion de documents scientifiques de niveau recherche, publiés ou non, émanant des établissements d'enseignement et de recherche français ou étrangers, des laboratoires publics ou privés. 
INRA Prod. Anim., $1995,8(4), 239-250$

\section{F. LABROUE}

INRA Station de Génétique quantitative et appliquée, 78352 Jouy-en-Josas Cedex
Facteurs de variation génétiques de la prise alimentaire chez le porc en croissance : le point des connaissances

Du fait de la sélection, on assiste à une évolution des types génétiques vers des animaux plus maigres, à croissance plus rapide et consommant moins d'aliment, donc à appétit plus faible. Une opinion largement partagée est que l'appétit risque de devenir, à long terme, un facteur limitant dans l'expression du potentiel de croissance du porc. Dans un contexte de sélection en alimentation à volonté - sans doute le mode d'alimentation des élevages de demain - il s'avère nécessaire d'étudier la possibilité d'introduire la capacité d'ingestion volontaire, ou un autre critère relatif au comportement alimentaire, parmi les objectifs de sélection. Pour cela, il est indispensable de bien connaître les différents facteurs génétiques qui influencent ce caractère dans les populations porcines.

Ces trente dernières années, la sélection des principales races porcines a porté pour l'essentiel sur l'amélioration des performances du porc en croissance, la sélection pratiquée consistant principalement en une sélection contre l'adiposité et pour l'efficacité alimentaire (gain de poids vif / aliment consommé). Le progrès génétique annuel obtenu pour les caractères de croissance et de composition cor-

\section{Résumé}

L'objectif de cet article est d'examiner les différents facteurs génétiques qui influencent la prise alimentaire, avant d'étudier la possibilité d'inclure un critère de comportement alimentaire dans les objectifs de sélection. Le type génétique peut entraîner des variations importantes $(10$ à $20 \%)$ du niveau de consommation et influencer les caractéristiques (fréquence, taille, durée) des repas. Les valeurs d'hétérosis pour la consommation moyenne journalière (5 à $10 \%$ de la moyenne parentale) sont du même ordre de grandeur que celles rapportées pour la vitesse de croissance ou l'efficacité alimentaire. Les écarts de consommation entre les trois génotypes pour le gène Hal varient entre 5 et $15 \%$. La moyenne des estimées pour l'héritabilité de la consommation alimentaire en conditions réelles d'alimentation ad libitum est 0,32. Les corrélations génétiques moyennes entre la consommation moyenne journalière d'une part, le gain moyen quotidien, l'indice de consommation et le taux de muscle d'autre part, sont respectivement de l'ordre de $0,75,0,40$ et $-0,45$. Les répercussions économiques éventuelles de la prise en compte de l'appétit dans les objectifs de sélection sont discutées sur la base de ces paramètres génétiques moyens. porelle est généralement de l'ordre de 0,5 à $1,5 \%$ de la moyenne dans les populations porcines nationales (Sellier et Rothschild 1991). Le gain génétique annuel dépasse même souvent $2 \%$ de la moyenne pour un caractère composite comme l'efficacité de la transformation de l'aliment consommé en tissu maigre ("lean tissue food conversion »).

Du fait des problèmes pratiques qu'elle soulève et de son coût élevé, la mesure individuelle de l'efficacité alimentaire ou de son inverse, l'indice de consommation (aliment consommé / gain de poids vif), est rarement réalisée dans les programmes actuels de sélection. L'amélioration de l'efficacité alimentaire est obtenue indirectement en utilisant des indices de sélection visant à la fois à augmenter la vitesse de croissance et à réduire la teneur en gras de la carcasse, cette dernière étant prédite chez les candidats à la sélection par la mesure in vivo de l'épaisseur de gras dorsal.

Cette orientation générale de la sélection en faveur de porcs à croissance plus rapide, à carcasse plus riche en muscle et à efficacité alimentaire améliorée conduit à des réponses corrélatives défavorables sur au moins deux points : 
- une tendance à la dégradation de certaines composantes de la qualité du produit: qualités technologiques et sensorielles de la viande, qualités technologiques du gras (insaturation des dépôts, part moindre de la synthèse de novo des acides gras saturés à partir de l'amidon, dépôt direct d'acides gras insaturés d'origine alimentaire) ;

- une tendance à une diminution de l'appétit (formation de tissus maigres moins coûteux en énergie) chez le porc en croissance (Kanis 1988, Webb 1989).

La réponse corrélative négative sur l'appétit est d'autant plus faible que l'indice de sélection utilisé accorde plus de poids à la vitesse de croissance. En effet, en accordant plus de poids à la vitesse de croissance, on donne également plus de poids au dépôt de tissus gras, si bien que la quantité d'aliment ingérée est moins liée au dépôt de protéines et donc à la croissance pondérale. C'est l'une des explications que l'on peut donner à l'absence de réponse corrélative vraiment notable pour l'appétit trouvée dans plusieurs expériences de sélection (Sather et Fredeen 1978, Vangen 1980, Cleveland et al 1983, Ollivier 1986, Canon et al 1991).

La tendance à une diminution de l'appétit chez le porc en croissance a été mise en évidence tant dans le cadre d'expériences de sélection (McPhee 1981, Ellis et al 1983, Wood et al 1983, Smith et al 1991, Castell et Cliplef 1993, Cameron et Curran 1994b) que par l'analyse des évolutions génétiques du caractère dans des populations nationales en Grande-Bretagne (Mitchell et al 1982) et en Allemagne (Brandt 1987). En France, les estimations de progrès génétique sont en général importantes pour le gain moyen quotidien mais l'étude d'Ollivier et al (1991) montre une absence d'évolution génétique de la consommation moyenne journalière dans les races Large White et Landrace Français entre 1977 et 1987.

Le risque que l'appétit devienne un facteur limitant de l'expression du potentiel de croissance du porc peut se trouver aggravé avec la perspective d'une utilisation croissante de mâles entiers qui ont déjà tendance à consommer moins d'aliment que les autres types sexuels (Fowler et al 1981, Cole et Chadd 1989), ou encore avec l'utilisation possible de promoteurs de croissance, comme la pST qui provoque une augmentation de la vitesse de croissance mais une diminution de la consommation moyenne par jour (Bidanel et al 1991).

\section{1 / Définitions et facteurs de variation}

L'appétit (ou capacité d'ingestion volontaire) se définit comme la quantité d'aliment ingérée par l'animal pendant une période donnée durant laquelle il a un accès permanent et illimité à l'aliment et à l'eau. L'unité de mesure de l'appétit est la consommation moyenne journalière d'aliment (CMJ) dans des conditions connues et maîtrisées de mesure (intervalle de poids, d'âge, ...). Généralement, on préfêre parler du profil alimentaire de consommation qui représente la consommation d'aliment par jour en fonction de l'âge ou du poids vif. Le comportement alimentaire se définit comme un ensemble de critères qui décrivent l'acte alimentaire non seulement en terme de quantité d'aliment ingérée, mais aussi en terme de répartition des prises d'aliment dans le temps : on parle de séquence alimentaire à l'échelle d'une journée ( 24 heures). Les principaux critères descriptifs du comportement alimentaire sont la consommation moyenne par jour (CMJ), la durée de consommation par jour (DCJ), la vitesse d'ingestion (consommation / durée), le nombre de repas par jour (NRJ) et la consommation moyenne par repas (CMR). L'étude de ces différents critères, chez le porc en croissance, peut être effectuée soit en alimentation individuelle soit en condition d'alimentation collective avec un distributeur automatique d'aliment.

La consommation d'aliment est liée à la fois aux dépôts journaliers de protéines (ou de tissus maigres) et de lipides (ou de gras). La quantité d'aliment ingérée par jour peut donc s'exprimer en fonction de :

- la part nécessaire à l'entretien, proportionnelle au poids métabolique, c'est-à-dire au poids vif élevé à la puissance représentative du besoin d'entretien (chez le porc en croissance, le poids métabolique correspond au poids vif élevé à la puissance 0,6 )

- la part correspondant au dépôt de tissus maigres (muscles, peau, viscères, os...)

- la part correspondant au dépôt de tissus gras (le dépôt d'un gramme de tissu gras est environ trois fois plus coûteux en énergie, et donc en aliment, que le dépôt d'un gramme de tissu maigre).

Chez le porc nourri ad libitum avec un régime non limitant en protéines, l'utilisation de l'énergie pour le dépôt de lipides ou de protéines est principalement déterminée par la relation entre l'énergie ingérée et la quantité de protéines déposées (ARC 1981). En première approximation, cette relation est de type " linéaire-en plateau " (Whittemore et Fawcett 1976, Kanis et al 1990 (figure 1). La quantité de protéines déposées $(\mathrm{Pd})$ augmente linéairement en fonction de l'énergie ingérée jusqu'à un plateau ( $\mathrm{Pd} \max$ ) représentant la vitesse maximale de dépôt de protéines de l'animal. Au-delà, l'excédent d'énergie ingérée est fixé sous forme de gras. Selon ce modèle, le but de la sélection est d'obtenir des animaux aussi proches que possible du point « CMJo», qui représente le minimum de consommation permettant d'obtenir la croissance musculaire maximale. Ce type de modèle suppose que le rapport gras / maigre dans la partie linéaire reste constant jusqu'au plateau. Cette simplification est désormais infirmée par l'expérimentation : le rapport gras / maigre dépend à la fois du poids vif de l'animal et du niveau d'énergie ingérée (de Greef et al 1994). Les mécanismes qui 


\section{Les distributeurs automatiques d'aliment}

Les distributeurs automatiques d'aliment souvent désignés sous le terme anglais de «CFIR (Computerized Food Intake Recording) systems " - ont été développés pour permettre la mesure de la consommation alimentaire individuelle de porcs en croissance élevés en loges collectives en condition d'alimentation à volonté. II en existe plusieurs types différents fabriqués aux Pays Bas système IVOG (Individual VOluntary food intake recording in Group housing) - ou en Grande Bretagne (système Fire Hunday). En France, les automates de distribution “Acema-48 » ont été conçus conjointement par le société Acemo de Pontivy (56) et le Cemagref (voir figure d'après Labroue et al 1994).

Chaque automate se compose d'une auge sur jauge de contrainte pouvant contenir jusqu'à $1,6 \mathrm{~kg}$ d'aliment en granulés, et d'un volet d'accès à l'auge permettant de détecter le début et la fin de la visite alimentaire. $\mathrm{Ce}$ volet est muni d'une antenne servant à lire le numéro d'identification de l'animal. L'auge est pesée au début et à la fin de chaque visite avec une précision de $0,5 \%$; elle est remplie à nouveau dès que le poids de l'aliment est inférieur à $400 \mathrm{~g}$.

Chaque animal porte à l'oreille une bague contenant une puce électronique protégée par une enveloppe plastique ; cette puce peut être lue dans un rayon de $35 \mathrm{~cm}$. A l'extrémité du couloir d'accès à l'auge, la porte arrière se bloque dès l'ouverture du volet et se débloque à sa fermeture, afin que l'animal ne soit pas dérangé lorsqu'il a accès à l'ali- ment. L'eau n'est disponible qu'à l'extérieur des automates afin de ne pas perturber les pesées d'aliment. Chaque distributeur automatique d'aliment peut accueillir jusqu'à 15 animaux entre 25 et $110 \mathrm{~kg}$ de poids vif.

Après chaque visite, le numéro de l'animal, l'heure de début et de fin de visite et la quantité d'aliment consommé sont enregistrés. La définition d'une visite est fortement liée au mode de fonctionnement du distributeur d'aliment. Chaque fois qu'un animal laisse retomber le volet de l'auge, une visite est enregistrée. Ainsi, le même repas d'un animal donné peut se trouver séparé en différentes visites successives. Pour permettre la comparaison des résultats avec ceux obtenus dans des conditions variées d'alimentation, les visites successives d'un même animal doivent être regroupées en un seul repas à l'aide du «critère de repas ". Ce critère se définit comme la durée maximale séparant deux visites à l'intérieur d'un même repas. Ainsi, si l'intervalle entre deux visites est supérieur à cette valeur, on en déduit qu'elles ne font pas partie du même repas. Les valeurs du critère de repas calculées chez des porcs en croissance varient entre 2 minutes (Labroue et al 1994) et 5 minutes (De Haer et Merks 1992).

Automate de distribution d'aliment « Acema48 (vu de dessus).

1 porte arrière, 2 couloir d'accès à l'auge, 3 bât-flanc articulé, 4 volet d'accès à l'auge, 5 auge, 6 dispositif de remplissage de l'auge.

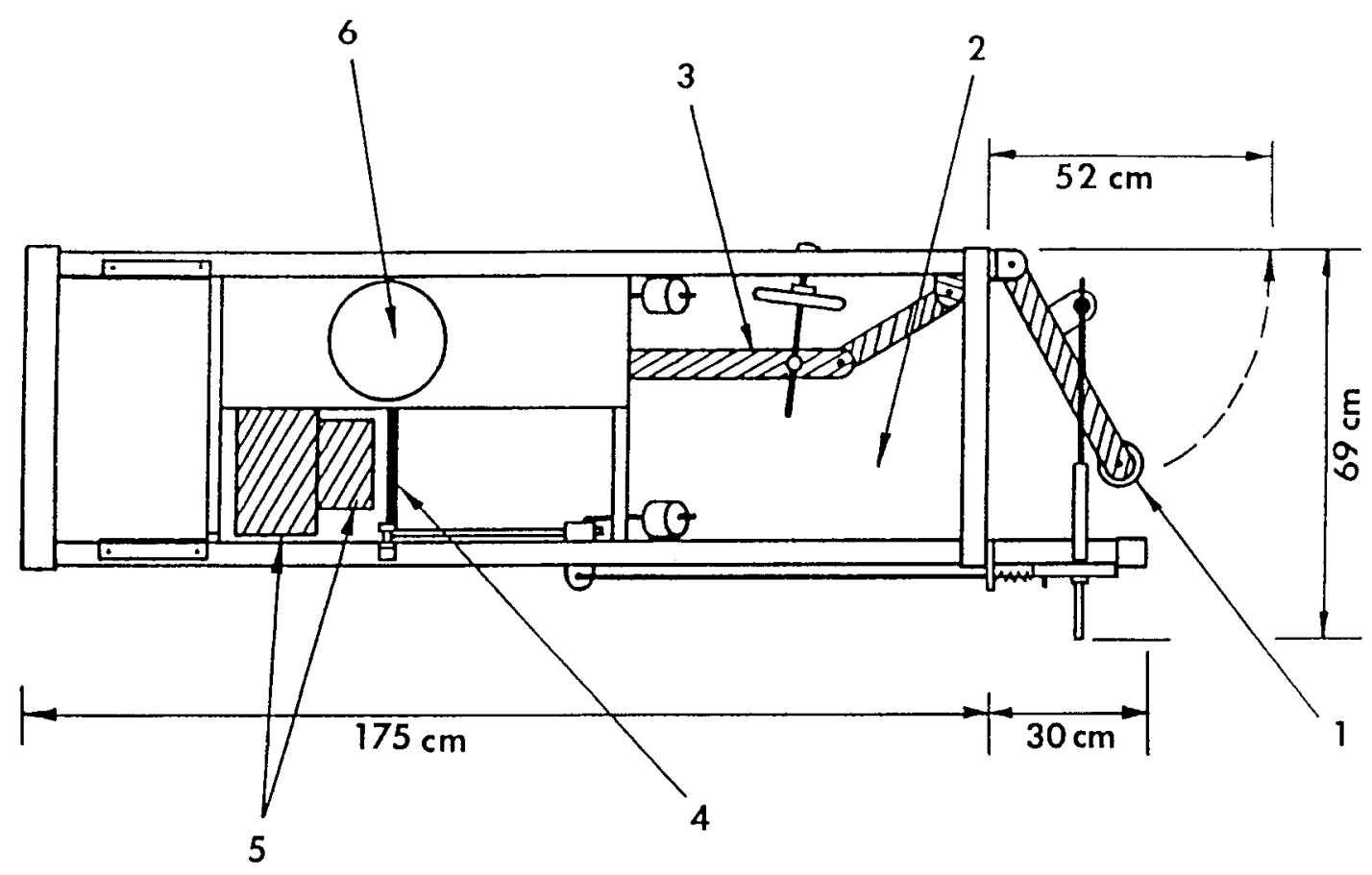


Figure 1. Relation « linéaire-en plateau » entre la quantité de protéines déposées et la consommation moyenne journalière d'aliment. E représente le niveau de consommation pour lequel on n'observe aucun dépôt de protéines. $C M J_{0}$ représente le niveau de consommation minimal pour réaliser le dépôt maximal de protéines (Pd max) (d'après Kanis et al 1990).

Protéines déposées

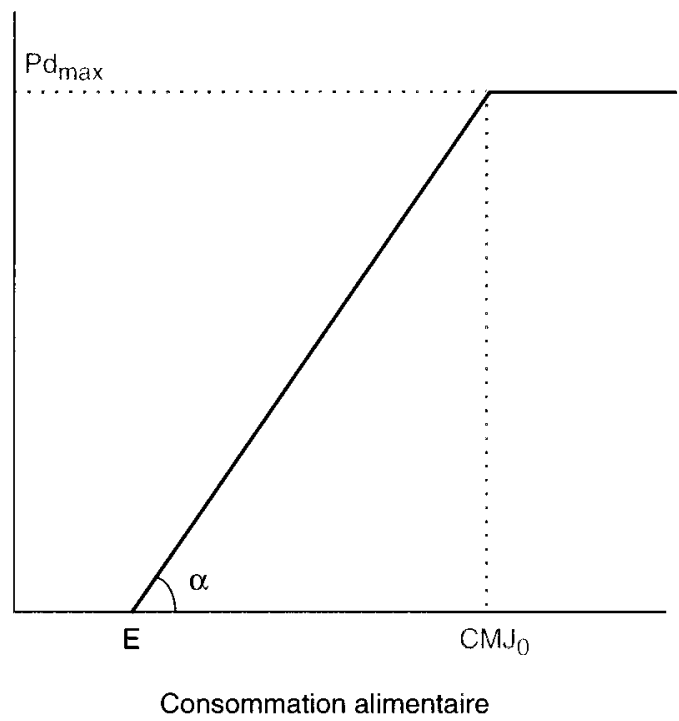

gouvernent l'utilisation de l'énergie pour les dépôts de lipides et de protéines durant la phase linéaire (avant d'atteindre la vitesse maximale de dépôt des protéines) doivent être définis de façon plus précise si l'on souhaite améliorer la prédiction des modèles de croissance qui utilisent le concept de la relation "linéaire-en plateau ". De plus, Campbell et Taverner (1988) n'observent pas systématiquement l'apparition d'un plateau pour la relation entre énergie ingérée et quantité de protéines déposées.

Différents facteurs influencent la prise alimentaire du porc en croissance, parmi lesquels ceux liés à l'animal - ses caractéristiques (race, type sexuel, âge, poids vif) et sa régulation physiologique, les facteurs extrinsèques, incluant l'alimentation et l'environnement tant social que physique, et, enfin, le déterminisme génétique. Cet article traite uniquement des facteurs de variation génétiques de la prise d'aliment, et s'articule autour de 4 thèmes principaux : les différences entre races, l'effet d'hétérosis, l'effet des gènes majeurs et les paramètres génétiques (héritabilités, corrélations phénotypiques et génétiques).

\section{2 / Différences entre races}

\section{1 / Consommation et efficacité alimentaire}

La consommation journalière d'aliment présente d'importantes variations aussi bien intra-race qu'entre races. Nous reviendrons plus loin sur la variabilité génétique intrarace. Chez les races Duroc, Yorkshire et Hampshire, plusieurs auteurs rapportent une croissance plus rapide $(+4,5 \%)$ et une consommation plus élevée $(+8 \%)$ pour la race Duroc tandis que l'efficacité alimentaire de la race Yorkshire est supérieure de $4 \%$ (Johnson et al 1973, Young et al 1976, Wilson et Johnson 1981). Les efficacités alimentaires des races Yorkshire et Poland China sont similaires (Kuhlers et al 1972, 1977) alors que la consommation moyenne journalière de la race Yorkshire semble légèrement plus élevée (Kuhlers et al 1972). Dans le cas des races porcines principalement utilisées en France, différents auteurs rapportent une consommation des porcs de race Piétrain inférieure de 10 à $20 \%$ à celle des animaux Landrace Français (Sellier et al 1974) ou Large White (Krieter et Kalm 1989, Webb 1989). De plus, l'écart de consommation observé entre races est très voisin pour les trois types sexuels (figure 2). Sellier et al (1974) ont comparé le niveau d'énergie ingérée journellement de 3 races : une race traditionnelle (Landrace Français) avec un taux de muscle standard et 2 races de type maigre, l'une à croissance rapide (Landrace Belge) l'autre à croissance lente (Piétrain). Les résultats montrent que, pour des gains de poids similaires, plus le rapport muscle sur gras est élevé et plus le niveau d'énergie consommée

Figure 2. Comparaison des consommations moyennes journalières entre 35 et $105 \mathrm{~kg}$ de poids vif pour des porcs de 3 races (Large White, Landrace Français ou Piétrain) et 3 types sexuels (mâles castrés, femelles, mâles entiers) (d'après Guéblez 1993, résultats non publiés).

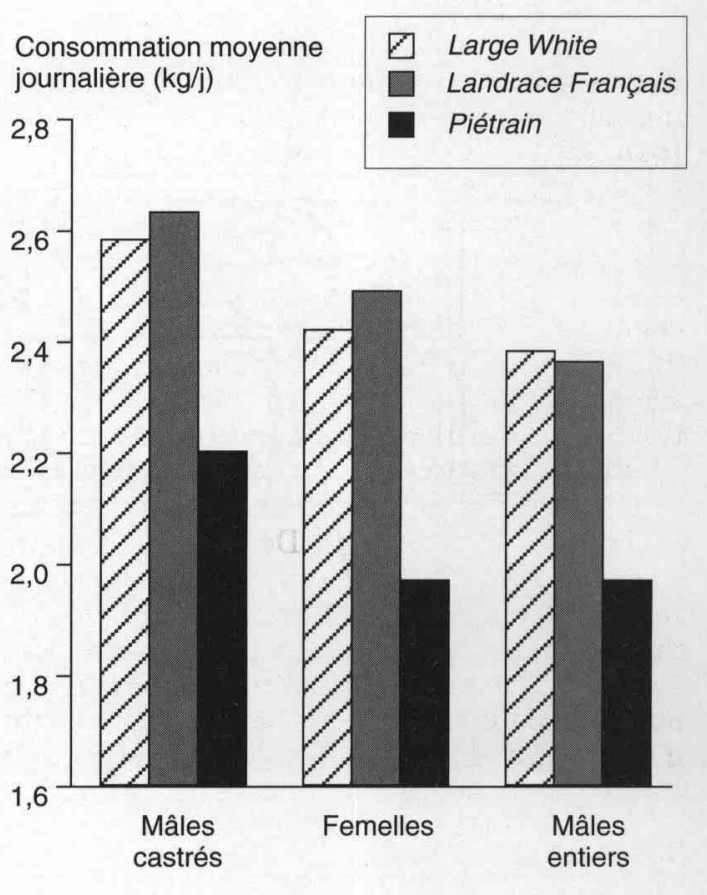


par jour est faible ; ceci traduit une meilleure efficacité alimentaire des animaux de type maigre. L'ajustement de l'appétit à un niveau moins élevé chez les porcs de type maigre s'explique simplement par un coût énergétique de ce gain plus faible. A l'opposé, une race de type gras comme la race Meishan présente une vitesse de croissance et une efficacité alimentaire moins élevées que celles des races Piétrain (Bidanel et al 1991) ou Large White (Haley et al 1992). La consommation moyenne journalière de la race Meishan est de l'ordre de 0,93 fois celle de la race Piétrain (Bidanel et al 1991). Selon Haley et al (1992), la consommation moyenne journalière de la race Meishan est toujours inférieure à celle de la race Large White mais les écarts de consommation observés varient avec le type sexuel : ils sont de l'ordre de $10 \%$ chez les femelles et de $30 \%$ chez les mâles entiers.

\section{2 / Comportement alimentaire}

Cet article s'appuie essentiellement sur des études réalisées en condition d'élevage en groupe à l'aide de distributeurs automatiques d'aliment. Labroue et al (1994) montrent que les différences entre les races Large White et Landrace Français concernent essentiellement la séquence alimentaire et non la quantité totale d'aliment ingérée : le nombre total de prises alimentaires est deux fois plus élevé pour les porcs Large White que pour les porcs Landrace Français. De Haer et Merks (1992) ont observé les mêmes écarts entre les animaux Landrace Néerlandais et Large White (Great Yorkshire), avec également une vitesse d'ingestion plus élevée chez ces derniers. Pour tous les critères relatifs au repas (nombre, durée et taille des repas), Labroue et al (1994) notent une interaction significative entre la race et le mode de regroupement par case (une seule race ou deux races mélangées). Dans les cases "monoraciales", les variables par repas ne présentent aucune différence entre races. En revanche, dans les cases mixtes, les animaux Landrace Français semblent modifier leur comportement alimentaire : ils effectuent moins de repas par jour mais ces repas sont plus longs et de taille plus importante que ceux des animaux Landrace Français en cases "monoraciales ». Ce changement de comportement est vraisemblablement le reflet de phénomènes de compétition plus intenses. Selon Labroue et al (1995), les mâles entiers Piétrain ont un comportement alimentaire sensiblement différent de celui des mâles entiers Large White. Ils effectuent moins de repas par jour $(5,1$ contre 7,0$)$ et leur vitesse d'ingestion est inférieure $(-8,9 \mathrm{~g} / \mathrm{min})$. De plus, l'écart de consommation observé entre ces races résulte principalement d'une différence de consommation en période diurne ( 8 h 00-20 h 00). Enfin, les animaux Piétrain présentent une activité alimentaire nocturne plus importante que les animaux Large White, aussi bien en condition d'alimentation collective (Labroue et al 1995) qu'en loges individuelles (Le Cozler 1994).

\section{3 / Effet d'hétérosis}

De nombreux auteurs (Sellier 1976, Johnson 1981, Schneider et al 1982, Mc Laren et al 1987) rapportent des valeurs d'hétérosis positives, de l'ordre de 6 à $10 \%$ de la moyenne des races parentales pour les performances de croissance (gain moyen quotidien ou âge à $100 \mathrm{~kg}$ de poids vif) et l'efficacité alimentaire (gain de poids vif / aliment consommé) pour des croisements faisant intervenir une grande variété de races porcines: Duroc, Hampshire, Yorkshire (Large White), Landrace, Piétrain, Poland China, Chester White, Spotted. Plusieurs études fournissent également des valeurs d'hétérosis pour la consommation moyenne journalière, estimée soit individuellement (Kuhlers et al 1972, 1977) soit par loges de 4 à 20 animaux (Johnson et al 1973, Young et al 1976, Wilson et Johnson 1981, Bidanel et al 1991, Haley et al 1992). A l'exception de Kuhlers et al (1972), tous ces auteurs rapportent une valeur d'hétérosis positive pour la consommation moyenne journalière, et ce quelles que soient les races considérées. Les valeurs sont de l'ordre de $0,10 \mathrm{~kg} / \mathrm{j}$ (soit $4,5 \%$ de la moyenne parentale) pour des croisements entre les races Duroc, Yorkshire et Hampshire (Johnson et al 1973, Young et al 1976), la valeur d'hétérosis du croisement DurocHampshire (environ 6,5\%) étant toujours supérieure à celle des croisements DurocYorkshire et Hampshire-Yorkshire. Selon Kuhlers et al (1977), l'effet d'hétérosis du croisement Poland China-Yorkshire est de $0,12 \mathrm{~kg} / \mathrm{j}(5,3 \%)$ supplémentaire pour la consommation moyenne journalière. Pour les croisements faisant intervenir la race chinoise Meishan, l'effet d'hétérosis sur la consommation moyenne journalière est encore plus important. Haley et al (1992) rapportent une valeur d'hétérosis de $0,19 \mathrm{~kg} / \mathrm{j}$ (soit $10 \%$ de la moyenne parentale) pour le croisement Meishan-Large White. Bidanel et al (1991) montrent que les croisés MeishanPiétrain consomment davantage d'aliment $(+0,73 \mathrm{~kg} / \mathrm{j})$ que les porcs Meishan, les animaux Piétrain ayant une consommation moyenne intermédiaire de l'ordre de $2,24 \mathrm{~kg} / \mathrm{j}$.

\section{4 / Gènes majeurs}

L'étude des effets du gène de la sensibilité à l'halothane - locus Hal, avec deux allèles : N (normal) et $n$ (sensible) - sur les performances du porc en croissance a commencé dès le début des années 1970. Webb (1989) suggère qu'une partie de la baisse de consommation observé dans la race Piétrain est due au gène de la sensibilité à l'halothane (n), qui est présent à une fréquence très élevée chez le Piétrain. Cette baisse de consommation est à mettre en relation avec un gain de muscle plus élevé.

Mc Phee et al (1994) ont étudié plus précisément l'effet du gène de la sensibilité à l'ha-

\section{Pour la consommation quotidienne, les valeurs d'hétérosis sont de l'ordre de $+6 \%$, quelles que soient les races parentales.}


lothane sur le niveau de consommation entre les poids de 25 et $90 \mathrm{~kg}$. Ils rapportent un écart de consommation de $0,36 \mathrm{~kg} / \mathrm{j}$, soit environ 1,5 écart-type du caractère, entre les deux génotypes homozygotes $\mathrm{NN}$ et $\mathrm{nn}$. Ils observent également un effet à peu près additif du gène $\mathrm{n}$ sur la consommation moyenne par jour : $2,46 \mathrm{~kg} / \mathrm{j}, 2,31 \mathrm{~kg} / \mathrm{j}$ et $2,10 \mathrm{~kg} / \mathrm{j}$ en moyenne respectivement pour les animaux $\mathrm{NN}, \mathrm{Nn}$ et $\mathrm{nn}$. Sur cette base, en admettant en première approximation que le gène n est fixé chez le Piétrain et que le gène $\mathrm{N}$ est fixé chez le Large White (Amigues et al 1994), la différence entre les animaux Piétrain (nn) et Large White (NN) s'expliquerait presque entièrement par l'effet du locus Hal (nn = $0,85 \mathrm{NN}$ ). Toutefois, Guéblez et al (1995) rapportent des différences nettement moins importantes entre les génotypes halothane (écart de $5 \%$ entre $\mathrm{NN}$ et $\mathrm{nn}$, avec $\mathrm{Nn}$ très voisin de $\mathrm{NN}$ )

La liste des gènes majeurs connus chez le porc ne se limite pas au gène de la sensibilité à l'halothane puisque l'existence du locus RN à effet majeur sur la qualité technologique de la viande de porc est désormais confirmée (Le Roy et al 1995). Des études récentes ont révélé la ségrégation de deux allèles au locus $\mathrm{RN}$ : un allèle récessif $\left(\mathbf{r n}^{+}\right)$normal, et un allèle dominant (RN) affectant de façon défavorable la qualité de la viande de porc. L'effet de ce locus sur les autres caractères de production est encore assez mal connu. Les premiers résultats obtenus dans ce domaine (Le Roy et al 1995) mettent en évidence une tendance à une efficacité alimentaire moindre de l'homozygote $\mathrm{rn}^{+} / \mathrm{rn}^{+}$par rapport aux deux autres génotypes. De plus, la vitesse de croissance de l'homozygote $\mathrm{rn}^{+} / \mathrm{rn}^{+}(860 \mathrm{~g} / \mathrm{j})$ est significativement plus faible que celle de l'hé- térozygote $(908 \mathrm{~g} / \mathrm{j})$, les homozygotes $\mathrm{RN}-\mathrm{RN}$ se trouvant en situation intermédiaire $(878 \mathrm{~g} / \mathrm{j})$

\section{5 / Paramètres génétiques}

L'introduction de l'appétit dans les stratégies et objectifs de sélection impose de bien connaître les paramètres génétiques de la consommation moyenne journalière (CMJ) et des critères du comportement alimentaire du porc en croissance : héritabilités $\left(h^{2}\right)$, corrélations phénotypiques $\left(r_{p}\right)$ et génétiques $\left(r_{A}\right)$ avec les autres caractères de production sélectionnés.

\section{1 / Héritabilités}

Le tableau 1 recense différentes estimations de l'héritabilité de la CMJ, obtenues en réelle situation d'alimentation à volonté, entre 1979 et 1994. Les valeurs de l'héritabilité de la CMJ s'échelonnent entre 0,16 et 0,62 , la moyenne des 16 estimées étant de 0,32 .

En plus de ces estimations obtenues en alimentation à volonté, on peut citer les résultats d'études conduites dans différents systèmes d'alimentation "semi à volonté" (1 ou 2 repas de durée limitée par jour, avec une quantité d'aliment offert ajustée régulièrement "selon l'appétit "). Dans ces études (Vangen 1980, Foster et al 1983, Standal et Vangen 1985, Johansson et al 1987), réalisées avec des animaux de race Large White (Yorkshire) ou Landrace, les estimées de l'héritabilité de la CMJ sont, à l'exception de l'une d'entre elles, inférieures à 0,20 . L'héritabilité

Tableau 1. Récapitulatif des estimations de l'héritabilité $\left(h^{2}\right)$ de la CMJ obtenues dans 13 études conduites en réelles conditions d'alimentation à volonté.

\begin{tabular}{|l|c|c|c|c|}
\hline \multicolumn{1}{|c|}{ Référence $^{(1)}$} & Races $^{(2)}$ & Types sexuels $^{(3)}$ & Période de contrôle & $\mathrm{h}^{2(4)}$ \\
\hline Mc Phee et al 1979 & LW, LR & ME & $25-80 \mathrm{~kg}$ & $0,62(0,13)$ \\
Wyllie et al 1979 & LW & ME & $23-118 \mathrm{~kg}$ & $0,23(0,09)$ \\
Valle-Zarate et Rogdakis 1980 & LR & ME, F & $30-85 \mathrm{~kg}$ & $0,36(0,10)$ \\
& LR & F & $30-100 \mathrm{~kg}$ & $0,54(0,19)$ \\
Blum 1983 & Y & F, MC & $25-103 \mathrm{~kg}$ & $0,23(0,05)$ \\
& LR & F, MC & $25-103 \mathrm{~kg}$ & $0,15(0,06)$ \\
Brandt 1987 & LR & F & & 0,50 \\
Cameron et al 1988 & LW & ME, F & $30-85 \mathrm{~kg}$ & $0,17(0,18)$ \\
& LR & ME, F & $30-85 \mathrm{~kg}$ & $0,26(0,17)$ \\
Van Steenbergen et al 1990 & LR, Y & ME & $25-105 \mathrm{~kg}$ & $0,24(0,06)$ \\
Cameron et al 1990 & LW, LR & ME & $30-91 \mathrm{~kg}$ & $0,34(0,08)$ \\
De Haer et de Vries 1993 & LR, Y & ME, F & $27-100 \mathrm{~kg}$ & $0,16(0,16)$ \\
Mrode et Kennedy 1993 & Y, LR, D & ME & $30-90 \mathrm{~kg}$ & $0,45(0,05)$ \\
De Vries et al 1994 & Y & ME, F, MC & $23-103 \mathrm{~kg}$ & $0,40(0,07)$ \\
Cameron 1994 & LW & ME, F & $30-85 \mathrm{~kg}$ & $0,29(0,03)$ \\
Cameron et Curran 1994a & LR & ME, F & $30-85 \mathrm{~kg}$ & $0,18(0,03)$ \\
\hline
\end{tabular}

(1) animaux élevés en groupes de 8 avec automate d'alimentation dans l'étude de De Haer et De Vries (1993), par groupes de 4 ( 2 femelles et 2 mâles castrés de la même portée) dans l'étude de Blum (1983), par groupe de 2 frères ou sceurs de portée dans les études de Brandt (1987) et Mrode et Kennedy (1993), en loges individuelles dans les neuf autres études.

(2) LW : Large White, LR : Landrace,Y : Yorkshire, D : Duroc.

(3) ME : Mâle Entier, F : Femelle, MC : Mâle castré.

(4) entre parenthèses : erreur-standard de l'estimée 
de la CMJ semble donc nettement plus faible en alimentation semi ad libitum qu'en alimentation réellement ad libitum.

De Haer et de Vries (1993) ont également estimé les héritabilités des critères de comportement alimentaire. Les estimées, dont les erreurs standard sont assez élevées $(0,16$ à $0,24)$, se trouvent toutes comprises entre 0,24 et 0,49 . Les durées de consommation sont moyennement héritables $\left(\mathrm{h}^{2}=0,25\right.$ en moyenne), contrairement au nombre quotidien des prises alimentaires, assez fortement héritable $\left(h^{2}=0,38\right.$ pour le nombre de visites et $\mathrm{h}^{2}=0,45$ pour le nombre de repas). Enfin, les quantités consommées par visite ou par repas ont également des héritabilités relativement élevées (respectivement de 0,35 et 0,47 ), alors que, dans cette étude, l'héritabilité de la CMJ n'est que de 0,16.

\section{2 / Corrélations phénotypiques}

\section{a / Entre les différents critères de comportement alimentaire}

Les corrélations phénotypiques entre les différents critères de comportement alimentaire ont été estimées par Labroue et al (1994). Ces corrélations sont très élevées (de l'ordre de 0,80 en valeur absolue) entre la durée, la taille et le nombre de repas. Ainsi, les porcs effectuant les plus gros repas font également moins de repas et des repas plus longs. Il semblerait qu'il existe toute une gamme de profils alimentaires allant des "amateurs de festin " (quelques repas par jour mais de gros repas) aux "grignoteurs " (beaucoup de petits repas). La CMJ est liée à la consommation moyenne par repas $\left(\mathbf{r}_{\mathrm{P}}=\right.$ $0,42)$ et à la vitesse d'ingestion $\left(\mathbf{r}_{\mathrm{p}}=0,37\right)$. La liaison, significative mais faible, entre la CMJ et le nombre de visites $\left(r_{p}=-0,16\right)$ ou le nombre de repas $\left(r_{p}=-0,15\right)$, tendrait à indiquer que les animaux qui mangent le plus sont des " amateurs de festin " plutôt que des " grignoteurs".

\section{b / Avec les autres caractères de production (tableau 2)}

Les moyennes des différentes estimations sont : $r_{\mathrm{P}}=0,63$ entre la CMJ et le gain moyen quotidien (GMQ), $r_{P}=0,35$ entre la CMJ et l'indice de consommation (IC) et $r_{p}=0,33$ entre la CMJ et l'épaisseur de lard dorsal (ELD).

D'autres critères de comportement alimentaire que la CMJ peuvent également être liés aux performances de production. De Haer et al (1993) rapportent des corrélations positives relativement élevées avec les performances pour la taille moyenne des repas et la vitesse d'ingestion $\left(\mathrm{r}_{\mathrm{p}}=0,50\right.$ avec le gain moyen quotidien, $\mathrm{r}_{\mathrm{P}}=0,35$ avec l'épaisseur de lard dorsal). En revanche, ces mêmes performances de production sont liées négativement aux nombres quotidiens de repas $\left(\mathrm{r}_{\mathrm{P}}=-0,20\right)$

Tableau 2. Récapitulatif des corrélations phénotypiques $\left(r_{p}\right)$ et génétiques $\left(r_{A}\right)$ entre la consommation moyenne journalière (CMJ) et les autres caractères de production : gain moyen quotidien (GMQ), indice de consommation (IC) et épaisseur de lard dorsal (ELD). Synthèse des résultats de 13 études conduites en réelles conditions d'alimentation à volonté.

\begin{tabular}{|c|c|c|c|c|c|c|c|}
\hline \multirow[b]{2}{*}{ Référence $^{(1)}$} & & \multicolumn{2}{|c|}{ CMJ et GMQ } & \multicolumn{2}{|c|}{ CMJ et IC } & \multicolumn{2}{|c|}{ CMJ et ELD } \\
\hline & & $\mathrm{r}_{\mathrm{P}}$ & $r_{A}^{(2)}$ & $r_{P}$ & $\mathrm{r}_{\mathrm{A}}$ & $\mathrm{r}_{\mathrm{P}}$ & $\mathrm{r}_{\mathrm{A}}$ \\
\hline Mc Phee et al 1979 & & 0,57 & $0,83(0,05)$ & 0,58 & $0,81(0,06)$ & 0,22 & $0,59(0,10)$ \\
\hline Wyllie et al 1979 & & 0,63 & 0,89 & 0,48 & 0,24 & & \\
\hline Valle-Zarate et & (3) & 0,59 & $0,68(0,10)$ & 0,40 & $0,52(0,14)$ & & \\
\hline Rogdakis 1980 & (4) & 0,74 & $0,69(0,12)$ & 0,33 & $0,23(0,20)$ & & \\
\hline Blum 1983 & $\mathrm{Y}$ & 0,77 & 0,71 & 0,26 & 0,38 & 0,39 & 0,52 \\
\hline & $\mathrm{LR}$ & 0,74 & 0,72 & 0,16 & 0,13 & 0,24 & 0,32 \\
\hline Brandt 1987 & & 0,73 & 0,84 & 0,10 & 0,01 & 0,33 & 0,49 \\
\hline Cameron et al 1988 & LW & 0,70 & $0,69(0,22)$ & 0,36 & $0,11(0,40)$ & & \\
\hline & LR & 0,50 & $0,73(0,60)$ & 0,48 & $1,00(0,19)$ & & \\
\hline Van Steenbergen et al 1990 & & 0,62 & $0,57(0,14)$ & 0,39 & $0,33(0,14)$ & 0,37 & $0,39(0,14)$ \\
\hline Cameron et al 1990 & & 0,54 & $0,58(0,15)$ & 0,45 & $0,30(0,15)$ & 0,40 & $0,56(0,15)$ \\
\hline De Haer et al 1993 & & 0,47 & & & & 0,35 & \\
\hline Mrode et Kennedy 1993 & & 0,74 & $0,80(0,03)$ & & & 0,33 & $0,42(0,06)$ \\
\hline De Vries et al 1994 & & 0,73 & 0,75 & & & 0,42 & 0,48 \\
\hline Labroue et al 1994 & & 0,65 & & 0,16 & & 0,36 & \\
\hline Cameron et & $\mathrm{LW}$ & 0,64 & $0,76(0,12)$ & 0,38 & $0,14(0,13)$ & 0,39 & $0,51(0,13)$ \\
\hline Curran $1994 \mathrm{~b}$ & LR & 0,37 & $0,41(0,23)$ & 0,42 & $0,55(0,12)$ & 0,13 & $0,24(0,12)$ \\
\hline Moyenne & & 0,63 & 0,73 & 0,35 & 0,37 & $\mathbf{0 , 3 3}$ & 0,45 \\
\hline
\end{tabular}

La liaison génétique entre consommation et gain de poids est élevée et assez stable alors que la liaison avec l'indice de consommation est plus faible et très variable selon les études.

(1) voir tableau 1 pour les conditions de réalisation des études (race, sexe, période de contrôle).

(2) entre parenthèses : erreur-standard de l'estimée.

(3) $30-85 \mathrm{~kg}$; (4) : $30-100 \mathrm{~kg}$

Y : Yorkshire, LW : Large White, LR : Landrace. 
ou de visites $\left(\mathbf{r}_{\mathrm{P}}=-0,15\right)$. Selon Labroue et al (1994), la seule variable de comportement alimentaire (autre que la CMJ) présentant une liaison notable avec les performances de production serait la vitesse d'ingestion $\left(r_{\mathrm{P}}=0,20\right.$ avec le GMQ, $r_{P}=0,13$ avec l'épaisseur de lard dorsal).

\section{3 / Corrélations génétiques (tableau 2)}

Il est à noter que ces estimées, dont certaines sont entachées d'une forte imprécision, sont assez cohérentes entre elles pour la liaison génétique entre la $\mathrm{CMJ}$ et le gain moyen quotidien ou l'épaisseur de lard dorsal mais que, en revanche, elles sont très variables en ce qui concerne la liaison entre la CMJ et l'indice de consommation. Les moyennes des différentes estimations sont: $\mathbf{r}_{\mathrm{A}}=0,73$ entre la $\mathrm{CMJ}$ et le gain moyen quotidien, $\mathrm{r}_{\mathrm{A}}=0,37$ entre la CMJ et l'indice de consommation et $\mathrm{r}_{\mathrm{A}}=0,45$ entre la CMJ et l'épaisseur de lard dorsal.

Des corrélations génétiques positives entre la CMJ et le dépôt journalier de tissus maigres ("lean tissue growth rate") sont rapportées par Cameron (1994) et Cameron et Curran (1994a), mais elles restent assez faibles : $0,23 \pm 0,07 \mathrm{chez}$ le Large White et $0,13 \pm 0,10$ chez le Landrace; pour Blum (1983) elles sont un peu plus élevées : 0,25 chez le Large White et 0,36 chez le Landrace.

\section{4 / Paramètres génétiques moyens}

Les valeurs moyennes de l'ensemble des paramètres génétiques de la consommation moyenne journalière et des performances de production, dans le contexte d'une alimentation à volonté, sont résumées dans le tableau 3 . Ces valeurs moyennes ont été établies à partir de la présente revue bibliographique

Tableau 3. Tableau récapitulatif des paramètres génétiques de la consommation moyenne journalière (CMJ) et des autres caractères de production : gain moyen quotidien (GMQ), indice de consommation (IC) et taux de muscle (TM), dans les conditions d'une alimentation à volonté.

\begin{tabular}{|l|c|c|c|c|}
\hline & CMJ & GMQ & IC & TM \\
\hline CMJ & $\mathbf{0 , 3 0}$ & $\mathbf{0 , 7 5}$ & $\mathbf{0 , 4 0}$ & $\mathbf{- 0 , 4 5}$ \\
& 0,40 & 0,70 & 0 & $-0,20$ \\
& 0,30 & 0,70 & - & $-0,20$ \\
\hline \multirow{2}{*}{ GMQ } & $\mathbf{0 , 6 5}$ & $\mathbf{0 , 3 5}$ & $\mathbf{- 0 , 3 5}$ & $\mathbf{- 0 , 2 5}$ \\
& 0,60 & 0,30 & $-0,70$ & $-0,10$ \\
& 0,70 & 0,30 & - & $-0,10$ \\
\hline IC & $\mathbf{0 , 3 5}$ & $\mathbf{- 0 , 4 5}$ & $\mathbf{0 , 3 0}$ & $\mathbf{- 0 , 2 5}$ \\
& 0 & $-0,70$ & 0,30 & $-0,30$ \\
& - & - & - & - \\
\hline TM & $\mathbf{- 0 , 3 5}$ & $\mathbf{- 0 , 1 5}$ & $\mathbf{- 0 , 1 5}$ & $\mathbf{0 , 5 5}$ \\
& $-0,30$ & $-0,10$ & $-0,30$ & 0,50 \\
& $-0,20$ & $-0,10$ & - & 0,50 \\
\hline
\end{tabular}

et, pour les caractères autres que la CMJ, de la mise au point récente de Ducos (1994a). La comparaison entre les résultats du tableau 3 et ceux de deux revues bibliographiques antérieures (Kanis 1988, Webb 1989) amène les remarques suivantes :

- il existe un excellent accord sur les estimations des héritabilités ;

- la relation génétique entre le GMQ et l'IC est moins étroite que celle rapportée par Webb (1989): $\mathrm{r}_{\mathrm{A}}=-0,35$ au lieu de -0,70;

- les relations génétiques sont en revanche un peu plus étroites entre le taux de muscle et le GMQ ( $\mathbf{r}_{\mathrm{A}}=-0,25$ au lieu de $\left.-0,10\right)$ et entre le taux de muscle et la CMJ $\left(r_{\mathrm{A}}=-0,45\right.$ au lieu de $-0,20$ );

- et surtout, la présente revue indique l'existence d'une assez forte "opposition génétique " et non pas d'une indépendance génétique entre l'IC et la CMJ $\left(r_{A}=0,40 \mathrm{au}\right.$ lieu de $r_{A}=0$ ).

\section{5 / Proposition de nouvelles stratégies de sélection}

Dans l'optique des évolutions à moyen et long terme des programmes de sélection, un appétit insuffisant peut compromettre les progrès futurs que peut apporter la sélection, et ceci d'autant plus que l'alimentation à volonté se généralise comme «milieu de sélection » et que la même évolution est à prévoir dans le milieu de production (élevages commerciaux). On peut considérer que l'efficacité de la croissance ne peut plus être beaucoup augmentée par une réduction du dépôt de gras chez l'animal en croissance (on est proche aujourd'hui de la limite biologique de teneur minimum en gras dans les races porcines les plus largement utilisées) et que les gains d'efficacité ultérieurs proviendront avant tout de la réduction de la dépense d'entretien à travers une croissance musculaire plus rapide. Le progrès futur viendra donc d'une augmentation de la vitesse de croissance des tissus maigres, qui suppose une augmentation de l'appétit (Fowler et al 1976).

Afin de prévenir la diminution de la consommation alimentaire journalière, il existe 3 possibilités pour prendre en compte ce critère dans la stratégie de sélection :

- utiliser le modèle "biologique" proposé par Fowler et al (1976) et sélectionner pour la vitesse de croissance des tissus maigres ;

- inclure un critère de consommation alimentaire dans l'objectif et / ou l'indice de sélection sur les performances de production;

- augmenter la pondération accordée au gain moyen quotidien dans l'objectif de sélection.

Selon Brandt (1987), l'utilisation directe de la consommation alimentaire journalière dans un objectif de sélection est rendue difficile par le fait qu'il n'existe pas de poids économique à lui affecter. En revanche, suite au développement de modèles biologiques de croissance basés sur la relation « linéaire-en plateau " entre le dépôt de protéines et la 
consommation (Kanis et al 1990), un des objectifs est l'établissement d'indices de sélection basés sur des valeurs économiques accordées à la consommation alimentaire (de Vries et Kanis 1992). Cependant, la composante majeure de la consommation est fixée par les évolutions conjointes des vitesses de dépôt de tissus maigres et gras. Ceci donne à la CMJ une signification composite, qui rend difficile son utilisation dans un indice de sélection.

Le moyen le plus simple, en apparence, serait d'augmenter le poids accordé à la vitesse de croissance. Les corrélations génétiques obtenues (tableau 2) montrent clairement que GMQ, CMJ, IC et ELD sont liés positivement. Dans ce contexte, l'attribution d'un poids plus important au gain moyen quotidien se justifie, à condition de disposer en même temps d'un critère bien représentatif du dépôt journalier de protéines ou de tissus maigres. Une bonne approximation de la vitesse de croissance des tissus maigres peut être obtenue par le produit: GMQ $\times R_{100} \mathrm{x}$ $\mathrm{TM}_{100}$ (où $R_{100}$ est le rendement de carcasse mesuré à $100 \mathrm{~kg}$ de poids vif, et $\mathrm{TM}_{100}$ le pourcentage de tissus maigres de la carcasse mesuré à $100 \mathrm{~kg}$ de poids vif).
A partir des paramètres génétiques rapportés dans le tableau 3, on peut calculer les évolutions génétiques annuelles attendues pour chacun des caractères dans diverses situations de sélection, c'est-à-dire pour différentes pondérations économiques du GMQ et du taux de muscle (TM) tandis que la pondération économique de l'IC est maintenue constante et prise par convention égale à -1 . Les hypothèses de calcul sont une intensité de sélection égale à 1 et un intervalle de génération de 2 ans. La situation actuelle de sélection en France (Ducos : 1994b, poids de 0,6 pour le GMQ, -1 pour l'IC et 1,7 pour le TM) entraîne une évolution génétique annuelle attendue de la CMJ d'environ -0,09 écart type du caractère (figure 3 ). Cet écarttype étant de l'ordre de $200 \mathrm{~g} / \mathrm{j}$, la réponse de l'appétit à la sélection actuelle est une diminution annuelle de la consommation moyenne journalière de $17 \mathrm{~g} / \mathrm{j}$. Pour obtenir le maintien de la CMJ à son niveau actuel, et étant donné la pondération économique du TM, il faudrait tripler le coefficient de pondération économique du GMQ.

Un autre moyen de maintenir l'appétit à son niveau actuel est d'utiliser un indice com-

Figure 3. Evolutions génétiques annuelles attendues $\left(\Delta G_{a}\right)$ de la consommation moyenne journalière (CMJ) dans diverses situations de sélection sur un indice individuel combinant le gain moyen quotidien (GMQ), l'indice de consommation (IC) et le taux de muscle (TM). L'objectif global de sélection est :

$H=a_{1} G M Q+a_{2} I C+a_{3} T M$

avec: $G M Q, I C$ et TM exprimés en unités d'écart-type phénotypique

$a_{1}=$ coefficient de pondération économique du GMQ

$a_{2}=-1$ (base constante)

$a_{3}=$ coefficient de pondération économique du TM

Hypothèses : paramètres génétiques du tableau 3, intensité de sélection 1, intervalle de génération de 2 ans.

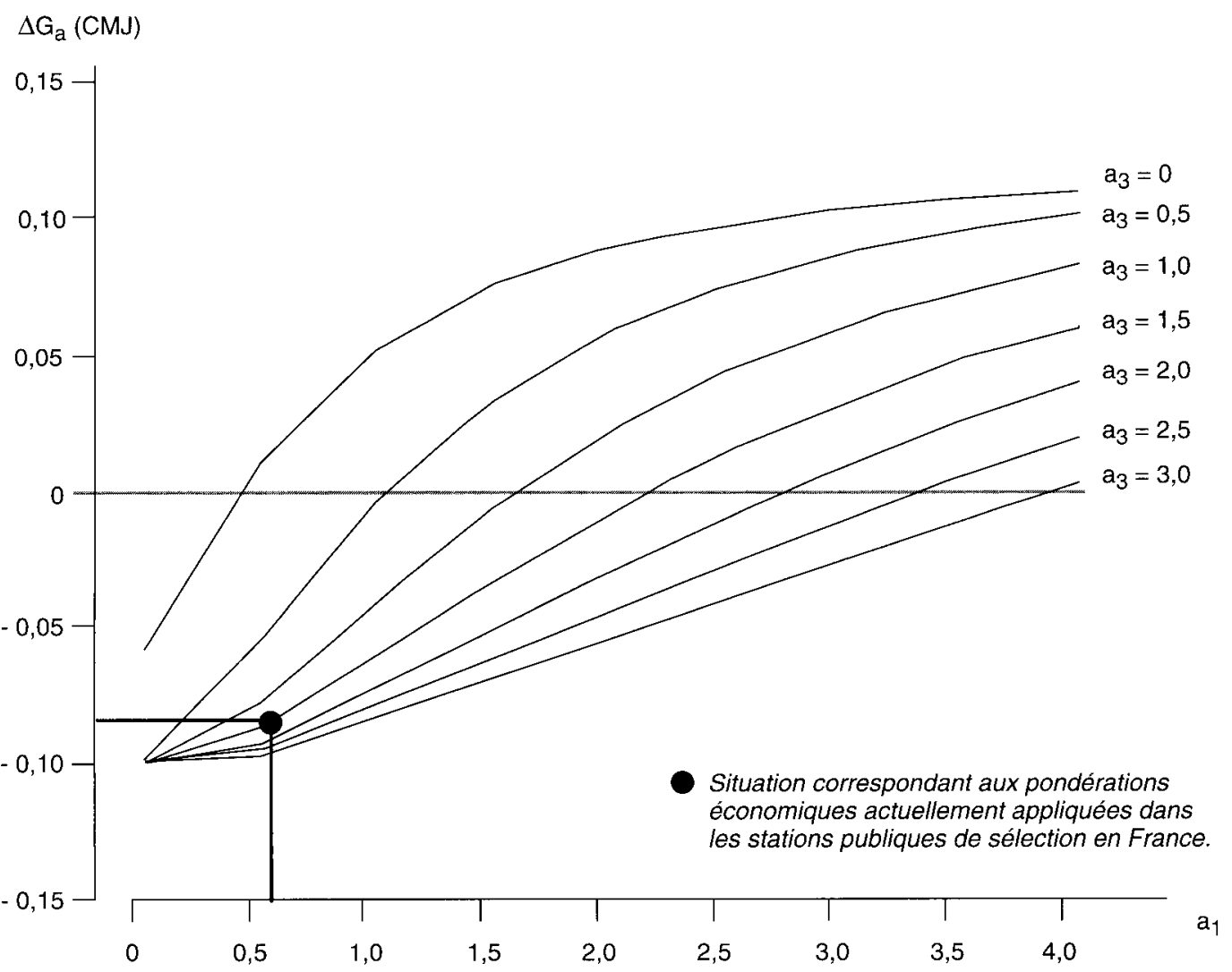


Tableau 4. Evolutions génétiques annuelles attendues pour les 3 caractères sélectionnés : gain moyen quotidien (GMQ), indice de consommation (IC) et taux de muscle (TM), et pour la consommation moyenne journalière (CMJ) dans diverses situations de sélection (hypothèses : voir figure 3).

\begin{tabular}{|cc|c|c|c|r|r|}
\hline & & \multicolumn{5}{|c|}{ Evolutions génétiques annuelles attendues } \\
$\mathrm{a}_{1}$ & $\mathrm{a}_{3}$ & $\mathrm{R}$ (Francs) & $\mathrm{GMQ}(\mathrm{g} / \mathrm{j})$ & \multicolumn{1}{|c|}{$\mathrm{IC}$} & $\mathrm{TM}(\%)$ & $\mathrm{CMJ}(\mathrm{g} / \mathrm{j})$ \\
\hline$* \mathbf{0 , 6}$ & $\mathbf{1 , 7}$ & $\mathbf{1 2 , 2 4}$ & $-\mathbf{0 , 8}$ & $\mathbf{- 0 , 0 2 1}$ & $\mathbf{0 , 8 4 0}$ & $-\mathbf{1 7 , 3}$ \\
1 & 0 & 4,14 & 12,4 & $-0,024$ & $-0,040$ & 10,5 \\
1 & 1 & 11,33 & 4,6 & $-0,025$ & 0,655 & $-8,3$ \\
1 & 2 & 12,20 & 0,3 & $-0,021$ & 0,822 & $-14,7$ \\
2 & 0 & 2,89 & 14,0 & $-0,018$ & $-0,112$ & 17,7 \\
2 & 1 & 8,91 & 9,6 & $-0,023$ & 0,404 & 4,4 \\
2 & 2 & 11,44 & 4,6 & $-0,022$ & 0,691 & $-5,9$ \\
3 & 0 & 2,38 & 14,5 & $-0,016$ & $-0,143$ & 20,7 \\
3 & 1 & 6,73 & 12,1 & $-0,020$ & 0,216 & 12,1 \\
3 & 2 & $\mathbf{1 0 , 0 0}$ & 7,9 & $-0,021$ & 0,536 & 1,9 \\
4 & 0 & 2,00 & 14,7 & $-0,014$ & $-0,159$ & 22,2 \\
4 & 1 & 5,22 & 13,3 & $-0,018$ & 0,092 & 16,5 \\
4 & 2 & 8,55 & 10,2 & $-0,020$ & 0,393 & 8,0 \\
\hline
\end{tabular}

$\mathrm{a}_{1}$ : coefficient de pondération économique du GMQ

$a_{2}$ : coefficient de pondération économique de l'IC constant $\left(a_{2}=-1\right)$

$a_{3}$ : coefficient de pondération économique du TM

* : situation de sélection actuelle

$\mathrm{R}$ : réponse globale pour la marge brute par porc

portant une contrainte restrictive pour la consommation alimentaire, et d'imposer ainsi l'absence d'évolution génétique pour la CMJ. Il est possible de chiffrer approximativement les répercussions économiques de l'utilisation de ce type d'indice. Si l'on se base sur les résultats du tableau 4 , une évolution génétique nulle de la $\mathrm{CMJ}$ correspondrait à une évolution génétique annuelle de la réponse globale $(R)$ pour la marge brute par porc d'environ 10,50 FF. Or, dans la situation actuelle, l'évolution génétique annuelle attendue pour $\mathrm{R}$ est de 12,24 FF. Autrement dit, l'utilisation d'un indice de sélection avec une contrainte restrictive sur la consommation alimentaire n'aurait pas de répercussions économiques très importantes puisque la diminution du progrès pour le taux de muscle serait compensée en grande partie par une forte aug- mentation du progrès pour le gain moyen quotidien.

Des études génétiques sont actuellement en cours sur divers critères de comportement alimentaire, chez des porcs en croissance élevés en groupe et alimentés à volonté à l'aide de distributeurs automatiques d'aliment. Ces études permettront de déterminer si, en plus ou au lieu de la consommation moyenne journalière, un critère de comportement alimentaire mérite d'être pris en compte dans les objectifs et / ou indices de sélection sur les performances du porc en croissance.

\section{Conclusion}

Le déterminisme génétique de la prise alimentaire chez le porc en croissance apparaît comme un phénomène relativement complexe. La race de l'animal est une source de variation importante à la fois pour le niveau de consommation et les critères de comportement alimentaire. Cet article souligne également l'existence de la variabilité induite par l'effet d'hétérosis ou l'effet de gènes majeurs. Avant d'inclure l'appétit (ou un autre critère de comportement alimentaire) dans les objectifs de sélection, il faudra aussi analyser la variabilité génétique intra-race des différents critères de comportement alimentaire, y compris sous l'angle de leurs relations génétiques avec les caractères usuels de croissance-carcasse. Une telle étude doit prendre en compte tous les autres facteurs (non génétiques) susceptibles d'influencer le comportement alimentaire. La connaissance des paramètres génétiques des différents critères de comportement alimentaire devrait ensuite permettre de choisir le (ou les) critère(s) à inclure dans les objectifs et / ou indices de sélection en vue d'améliorer le niveau de consommation, ou au moins, de le maintenir constant.

\section{Remerciements}

Ce travail bibliographique a été réalisé dans le cadre d'une bourse de thèse cofinancée par l'Institut Technique du Porc et l'INRA.

\section{Références bibliographiques}

Amigues Y., Runavot J.P., Sellier P., 1994. Evaluation de la fréquence du gène de sensibilité à l'halothane dans les principales races porcines françaises en 1993. Techni-Porc, 17 (3), 23-28.

ARC (Agricultural Research Council), 1981. The nutrient requirements of pigs. Commonwealth Agricultural Bureaux, Slough, U.K., 307 pp.

Bidanel J.P., Bonneau M., Pointillart A., Gruand J., Mourot J., Demade I., 1991. Effects of exogenous porcine somatotropin ( $\mathrm{pST}$ ) administration on growth performance, carcass traits, and pork meat quality of Meishan, Piétrain and crossbred gilts. J. Anim. Sci., 69, 3511-3522.
Blum J.K., 1983. Populationsanalyse der schweizerischen Schweinerassen. Diss., ${ }^{\circ} 7412$, E.T.H. Zürich, $152 \mathrm{pp}$.

Brandt H., 1987. Development and genetic aspects of feed intake in three breeds of pigs at German test stations and measures to prevent further deterioration. Pig News Inf., 8, 29-33.

Cameron N.D., 1994. Selection for components of efficient lean growth rate in pigs : 1 . Selection pressure applied and direct responses in a Large White herd. Anim. Prod., 59, 251-262.

Cameron N.D., Curran M.K., 1994a. Selection for components of efficient lean growth rate in pigs : 
2. Selection pressure applied and direct responses in a Landrace herd. Anim. Prod., 59, 263-269.

Cameron N.D., Curran M.K., 1994b. Selection for components of efficient lean growth rate in pigs : 4 . Genetic and phenotypic parameter estimates and correlated responses in performance test traits with ad libitum feeding. Anim. Prod., 59, 281-291.

Cameron N.D., Curran M.K., Thompson R., 1988. Estimation of sire with feeding regime interaction in pigs. Anim. Prod., 46, 87-95.

Cameron N.D, Pearson M., Richardson B., Brade H., 1990. Genetic and phenotypic parameters for performance traits in pigs with ad libitum and restricted feeding. Proceedings of the 4 th world congress on genetics applied to livestock production, Edinburgh, vol 15, 473-476.

Campbell R.G., Taverner M.R., 1988. Genotype and sex effects on the relationship between energy intake and protein deposition in growing pigs. J. Anim. Sci., 66, 676-686.

Canon J., Gruand J., Gutierrez J.P., Ollivier L., 1991. A repeat-sire mating experiment on lean tissue growth in the pig. 42nd Annual Meeting of the EAAP, Berlin, September 8-12, 1991.

Castell A.G., Cliplef R.L., 1993. Related responses in the live performance and carcass measurements of progeny from sows selected for thinner backfat. Can. J. Anim. Sci., 73, 471-482.

Cleveland E.R., Johnson R.K., Mandigo R.W., 1983. Index selection and feed intake restriction in swine. 1. Effect on rate and composition of growth. J. Anim. Sci., 56, 560-569.

Cole D.J.A., Chadd S.A., 1989. Voluntary food intake of growing pigs. In : Forbes J.M., Varley M.A., Laurence T.L.J. (Eds), The Voluntary Food Intake of pigs, B.S.A.P. Occasional Publication n ${ }^{\circ} 13$, 61-70.

Ducos A., 1994a. Paramètres génétiques des caractères de production chez le porc : mise au point bibliographique. Techni-Porc, 17 (3), 35-67.

Ducos A., 1994b. Evaluation génétique des porcs contrôlés dans les stations publiques à l'aide du BLUP - modèle animal. Performances et Sélection, 94-03, 1-9.

Ellis M., Smith W.C., Henderson R., Whittemore C.T., Laird R., 1983. Comparative performance and body composition of control and selection line in Large White pigs. 2. Feeding to appetite for a fixed time. Anim. Prod., 36, 407-413.

Foster W.H., Kilpatrick D.J., Heaney I.H., 1983. Genetic variation in the efficiency of energy utilization by the fattening pig. Anim. Prod., 37, 387-393.

Fowler V.R., Bichard M., Pease A.H.R., 1976. Objectives in pig breeding. Anim. Prod., 23, 365-387.

Fowler V.R., Mc William R., Aitken R., 1981. Voluntary feed intake of boars, castrates and gilts given diets of different nutrient density. Anim. Prod., 32, 357 (Abstr).

Greef K.H. (de), Verstegen M.W.A., Kemp B., van der Tog P.L., 1994. The effect of body weight and energy intake on the composition of deposited tissue in pigs. Anim. Prod., 58, 263-270.

Guéblez R., Pabœuf F., Sellier P., Bouffaud M., Boulard J., Brault D., Le Tiran M.H., Petit G., 1995. Effet du génotype halothane sur les performances d'engraissement, de carcasse et de qualité de viande du porc charcutier. Journées Rech. Porcine en France, 27, 155-164.

Haer L.C.M. (de), Merks J.W.M., 1992. Patterns of daily food intake in growing pigs. Anim. Prod., 54, 95-104.
Haer L.C.M. (de), Vries A.G. (de), 1993. Effects of genotype and sex on the feed intake pattern of group housed growing pigs. Livest. Prod. Sci., 36, 223-232.

Haer L.C.M. (de), Luiting P., Aarts H.L.M., 1993. Relations among individual (residual) feed intake, growth performance and feed intake pattern of growing pigs in group housing. Livest. Prod. Sci., 36, 233-253.

Haley C.S., d'Agaro E., Ellis M., 1992. Genetic components of growth and ultrasonic fat depth traits in Meishan and Large White pigs and their reciprocal crosses. Anim. Prod., 54, 105-115.

Johansson K., Andersson K., Lundeheim N., 1987. Evaluation of station testing of pigs. 1. Genetic parameters for feed measurements and selection effects on voluntary feed intake. Acta Agric. Scand., 37, 93-107.

Johnson R.K., 1981. Crossbreeding in swine : experimental results. J. Anim. Sci., 52, 906-923.

Johnson R.K., Omtvedt I.T., Walters L.E., 1973. Evaluation of purebreds and two-breed crosses in swine: feedlot performance and carcass merit. J. Anim. Sci., 37, 18-26.

Kanis E, 1988. Food intake capacity in relation to breeding and feeding of growing pigs. Thesis, Agricultural University, Wageningen, 139 pp.

Kanis E., Vries A.G. (de), Morel P.C.H., 1990. Genetic and economic aspects of appetite in growing pigs. 41st Annual Meeting of the E.A.A.P., P 2.1, Toulouse, July 9-12, 1990.

Krieter J., Kalm E., 1989. Growth, feed intake and mature size in Large White and Pietrain pigs. J. Anim. Breed. Genet., 106, 300-311.

Kuhlers D.L., Chapman A.B., First N.L., 1972. Estimates of genotype-environment interactions in production and carcass traits in swine. J. Anim. Sci., $35,1-7$.

Kuhlers D.L., Chapman A.B., First N.L., 1977. Estimates of genotype-environment interactions within and between two breeds of swine for production and carcass traits. J. Anim. Sci., 44, 549-556.

Labroue F., Guéblez R., Sellier P., Meunier-Salaün M.C., 1994. Feeding behaviour of group-housed Large White and Landrace pigs in French central test stations. Livest. Prod. Sci., 40, 303-312.

Labroue F., Guéblez R., Marion M., Sellier P., 1995. Influence de la race sur le comportement alimentaire de porcs en croissance élevés en groupe : premiers résultats d'une comparaison Large White-Piétrain. Journées Rech. Porcine en France, 27, 175-182.

Le Cozler Y., 1994. Production de chaleur et comportement alimentaire chez le porc en relation avec son potentiel de croissance. Diplôme de fin d'études, Université Paris 7, France, 32 pp.

Le Roy P., Caritez J.C., Billon Y., Elsen J.M., Talmant A., Vernin P., Lagant H., Larzul C., Monin G., Sellier P., 1995. Etude de l'effet du locus RN sur les caractères de croissance et de carcasse : premiers résultats. Journées Rech. Porcine en France, 27, 165-170.

Mc Laren D.G., Buchanan D.S., Johnson R.K., 1987. Individual heterosis and breed effects for postweaning performance and carcass traits in four breeds of swine. J. Anim. Sci., 64, 83-98.

Mc Phee C.P., 1981. Selection for efficient lean growth in a pig herd. Austr. J. Agric. Res., 32, 681690 . 
Mc Phee C.P., Brennan P.J., Duncalfe F., 1979. Genetic and phenotypic parameters of Australian Large White and Landrace boars performancetested when offered food ad libitum. Anim. Prod., 28, 79-85

Mc Phee C.P., Daniels L.J., Kramer H.L., Macbeth G.M., Noble J.W., 1994. The effects of selection for lean growth and the halothane allele on growth performance and mortality of pigs in a tropical environment. Livest. Prod. Sci., 38, 117-123.

Mitchell G., Smith C., Makower M., Bird P.J.W.N., 1982. An economic appraisal of pig improvement in Great Britain. 1. Genetic and production aspects. Anim. Prod., 35, 215-224.

Mrode R.A., Kennedy B.W., 1993. Genetic variation in measures of food efficiency in pigs and their genetic relationships with growth rate and backfat. Anim. Prod., 56, 225-232.

Ollivier L., 1986. Result of a long-term selection experiment for lean tissue growth in the pig. Proceedings of the 3rd world congress on genetics applied to livestock production, vol 12, 168-180.

Ollivier L., Lagant H., Gruand J., Molénat M., 1991. Progrès génétique des porcs Large White et Landrace Francais entre 1977 et 1987. Journées Rech. Porcine en France, 23, 389-394.

Sather A.P., Fredeen H.T., 1978. Effect of selection for lean growth rate upon feed utilisation by the market hog. Can. J. Anim. Sci., 58, 258-289.

Schneider J.F., Christian L.L., Kuhlers D.L., 1982. Crossbreeding in swine: genetic effects on pig growth and carcass merit. J. Anim. Sci., 54, 747 756.

Sellier P., 1976. The basis of crossbreeding in pigs : a review. Livest. Prod. Sci., 3, 203-226.

Sellier P., Rothschild M.F., 1991. Breed identification and development in pigs. In : Genetic resources of pig, sheep and goat, World Animal Science B8, 125-143. Elsevier Amsterdam, New York.

Sellier P., Houix Y., Desmoulin B., Henry Y., 1974. Premières observations sur la relation entre conditions nutritionnelles et type génétique chez des porcs femelles. Journées Rech. Porcine en France, 6, 209-219.

Smith W.C., Ellis M., Chadwick J.P., Laird R., 1991. The influence of index selection for improved growth and carcass characteristics on appetite in a population of Large White pigs. Anim. Prod., 52, 193-199.

Standal N., Vangen O., 1985. Genetic variation and covariation in voluntary food intake in pig selection programmes. Livest. Prod. Sci., 12, 367-377.

Steenbergen (van) E.J., Kanis E., van der Steen H.A.M., 1990. Genetic parameters of fattening performance and exterior traits of boars tested in central stations. Livest. Prod. Sci., 24, 65-82.

Valle-Zarate A., Rogdakis E., 1980. Populationsgenetische Parameter der Futteraufnahme beim Schwein. 31st Annual Meeting of EAAP, P 5/6.29, Munich, September 1-4, 1980.

Vangen O., 1980. Studies on a two trait selection experiment in pigs. 3 . Correlated responses in daily feed intake, feed conversion and carcass traits. Acta Agric. Scand., 30, 125-141.

Vries A.G. (de), Kanis E., 1992. A growth model to estimate economic values for food intake capacity in pigs. Anim. Prod., 55, 241-246.

Vries A.G. (de), van der Wal P.G., Long T., Eikelenboom G., Merks J.W.M., 1994. Genetic parameters of pork quality and production traits in Yorkshire populations. Livest. Prod. Sci., 40, 277-289.

Webb A.J., 1989. Genetics of food intake in the pig. In : Forbes J.M., Varley M.A., Laurence T.L.J. (Eds) The Voluntary Food Intake of pigs, B.S.A.P. Occasional Publication $n^{\circ} 13$, pp. 41-50.

Whittemore C.T., Fawcett R.H., 1976. Theoretical aspects of a flexible model to simulate protein and lipid growth in pigs. Anim Prod., 22, 87-96.

Wilson E.R., Johnson R.K., 1981. Comparison of three-breed and backcross swine for litter productivity and postweaning performance. J. Anim. Sci., $52,18-25$

Wood J.D., Whelehan O.P., Ellis M., Smith W.C., Laird R., 1983. Effects of selection for low backfat thickness in pigs on the sites of tissue deposition in the body. Anim. Prod., 36, 389-397.

Wyllie D., Morton J.R., Owen, J.B., 1979. Genetic aspects of voluntary food intake in the pig and their association with gain and food conversion efficiency. Anim. Prod., 28, 381-390.

Young L.D., Johnson R.K., Omtvedt I.T., Walters L.E., 1976. Postweaning performance and carcass merit of purebred and two-breed cross pigs. J. Anim. Sci., 42, 1124-1132

\begin{abstract}
Genetic factors determining food intake in growing pigs : a review.

The aim of this paper is to investigate the different genetic factors that influence feed intake, previously to study the possible inclusion of a feeding behaviour criterion in the selection objective. Breed is an important souce of varia. tion of feed intake (differences of 10 to $20 \%$ ). In addition, breed could influence meal characteristics (frequency, size, duration). Heterosis estimates for daily feed intake (5 to $10 \%$ of the parental mean) are similar to those reported for growth rate and feed efficiency. Feed intake differences between the three genotypes at the Hal
\end{abstract}

Abstract

locus range from 5 to $15 \%$. The average of estimates for feed intake heritability in real ad libitum feeding conditions is $\mathbf{0 . 3 2}$. The average genetic correlations between daily feed intake on the one hand, and average daily gain, food conversion ratio and carcass lean content on the other hand, are around $\mathbf{0 . 7 5}, 0.40$ and $\mathbf{- 0 . 4 5}$, respectively. The possible consequences of including appetite in the selection objective are discussed on the basis of these average genetic parameters.

LABROUE F., 1995. Facteurs de variation génétiques de la prise alimentaire chez le porc en croissance : le point des connaissances. INRA Prod. Anim., 8 (4), 239-250. 